\title{
Vector and tensor contributions to the curvature perturbation at second order
}

\section{Pedro Carrilho and Karim A. Malik}

Astronomy Unit, School of Physics and Astronomy, Queen Mary University of London, Mile End Road, London, E1 4NS, UK

E-mail: p.gregoriocarrilho@qmul.ac.uk,k.malik@qmul.ac.uk

\begin{abstract}
We derive the evolution equation for the second order curvature perturbation using standard techniques of cosmological perturbation theory. We do this for different definitions of the gauge invariant curvature perturbation, arising from different splits of the spatial metric, and compare the expressions. The results are valid at all scales and include all contributions from scalar, vector and tensor perturbations, as well as anisotropic stress, with all our results written purely in terms of gauge invariant quantities. Taking the largescale approximation, we find that a conserved quantity exists only if, in addition to the non-adiabatic pressure, the transverse traceless part of the anisotropic stress tensor is also negligible. We also find that the version of the gauge invariant curvature perturbation which is exactly conserved is the one defined with the determinant of the spatial part of the inverse metric.
\end{abstract}

Keywords: cosmological perturbation theory, physics of the early universe

ArXiv ePrint: 1507.06922 


\section{Contents}

1 Introduction $\quad 1$

2 Definitions and Conventions 2

2.1 Metric Tensor 3

2.2 Stress-energy Tensor $\quad 4$

2.3 The gauge problem 5

3 Gauge invariant quantities $\quad 6$

3.1 Curvature perturbation on uniform density hypersurfaces 6

$\begin{array}{lll}3.2 & \text { Non-adiabatic pressure } & 9\end{array}$

4 Evolution equations $\quad 9$

4.1 Fluid equations $\quad 9$

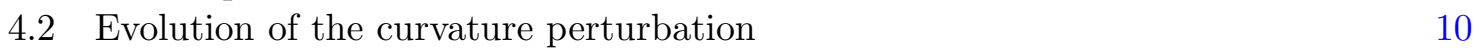

4.3 Large scale approximation 12

5 Conclusion 13

$\begin{array}{lr}\text { A Second order gauge transformations } & 14\end{array}$

B Large scale limit of the gauge invariant quantities $\quad 15$

$\begin{array}{lr}\text { C On intrinsic and extrinsic curvature } & 16\end{array}$

\section{Introduction}

Advances in observational cosmology in the last decades have provided us with countless ways to test cosmological models and so far, the Standard Model of Cosmology has passed all of them. In the simplest version this standard model of cosmology comprises a period of accelerated expansion, inflation, at the very beginning and has the dynamics of the universe dominated by dark matter, and later dark energy, after an initial epoch of radiation domination.

In recent years the results from the WMAP [1] and the Planck [2] missions have provided us with an unprecedented amount of information from the temperature anisotropies in the Cosmic Microwave Background (CMB), constraining the allowed parameter space. Also, recent B-mode polarisation measurements [3] have put additional tight bounds on the tensor-to-scalar ratio. In spite of all this progress, the correct model of the early Universe is still unknown, as there are many inflationary potentials that are consistent with the experimental results. Future surveys will provide an even further improvement in the experimental accuracy and should probe more scales than ever before. However, in order to make the most of these new observations, further efforts are required also on the theory side, for example to compute observables with the precision required by the experiments.

According to inflationary theory, the seeds of structure were generated during inflation through the quantum fluctuations of one or more light scalar fields. To study the evolution of these initial perturbations we use Cosmological Perturbation Theory. This amounts to 
adding inhomogeneous perturbations to the Friedmann-Lemaitre-Robertson-Walker (FLRW) solution and studying their evolution equations. While the linear theory seems to have been successful so far, higher order corrections seem necessary, in order to study new observables, such as non-gaussianities, and to make more accurate predictions of the observables we already measure.

The gauge invariant curvature perturbation on uniform density hypersurfaces, $\zeta$, is a key variable when computing predictions from inflation [4-7]. Its correlation functions encode the relevant information about the micro-physics of inflation and are also related with observables we can probe, such as the temperature anisotropies in the CMB and the distribution of galaxies in the sky. It is, therefore, fundamental to have a precise knowledge about the evolution of this curvature perturbation. It is well known that, on super-horizon scales and for adiabatic perturbations, $\zeta$ is conserved at first order (see e.g. Ref. [8]). This result has been extended to higher orders using non-perturbative methods such as the $\delta N$ formalism [9] and using other approaches (see e.g. Refs. [10, 11]). At second order in cosmological perturbation theory, the conservation of the curvature perturbation has been established for scalar perturbations in Ref. [12].

However, the evolution equation at all scales and at second order in the perturbative expansion including scalar, vector and tensor perturbations has so far not been fully explored ${ }^{1}$. Furthermore, at second order, there exist different definitions of this gauge invariant perturbation, and it is not clear that the conservation of one implies the conservation of the others.

In this work, we aim to address both of these issues. We start by reviewing the different versions of the gauge invariant curvature perturbation on uniform density hypersurfaces and show how they are related. We then derive the evolution equation for each convention and compare the results. Besides the scalar contributions we also keep all vector and tensor contributions, as well as the anisotropic stress. Finally, we take the large scale limit and check for the conditions of existence of conserved quantities.

The paper is structured as follows. In the next section, we present the different conventions for the metric perturbations and give the necessary gauge transformations. The different definitions of $\zeta$ are given in Section 3, along with a number of auxiliary gauge invariant quantities. A derivation of the evolution of $\zeta_{2}$ is presented in Section 4. We then present our conclusions in Section 5.

\section{Definitions and Conventions}

In this initial section, we introduce our conventions for the metric and stress-energy tensors. We simultaneously perform the $3+1$ decomposition of space-time and and also the scalarvector-tensor (SVT) decomposition, in which tensors in the spatial slicing are split into their scalar, divergence-free vector and transverse and traceless tensor parts according to their transformation behaviour on the 3-dimensional submanifolds. For the most part, we follow the notation of [17]. All quantities $T$ are expanded as

$$
T=T_{0}+\delta T_{1}+\frac{1}{2} \delta T_{2}+\ldots
$$

\footnotetext{
${ }^{1}$ Note, however, the early works of Tomita in Refs. [13-15] regarding the interaction between scalar, vector and tensor modes in the Einstein-de Sitter universe, as well as more recent work in Ref. [16] and references therein.
} 
in which the subscript denotes the order in perturbation theory. We will drop the subscript for the background quantities as the unperturbed ones only appear at their definition.

Throughout the paper we assume a FLRW background spacetime with zero spatial curvature, and use conformal time. Greek indices, $\mu, \nu, \lambda$, range, from $0, \ldots 3$, while lower case Latin indices, $i, j, k$, denote spatial indices ranging from $1, \ldots 3$.

\section{$2.1 \quad$ Metric Tensor}

The metric tensor can be split in several different ways. We will show four distinct ones, which vary in the way the spatial part of the metric is arranged. The version that we will use in most of the calculations below takes the following form, at all orders

$$
\begin{aligned}
g_{00} & =-a^{2}(1+2 \phi), \\
g_{i 0} & =a^{2}\left(B_{, i}-S_{i}\right), \\
g_{i j} & =a^{2}\left[\delta_{i j}+2 C_{i j}\right],
\end{aligned}
$$

in which $\phi$ is the perturbation to the lapse, $B$ and $S_{i}$ are, respectively, the scalar and vector parts of the shift and $C_{i j}$ is the perturbation to the spatial part of the metric. The first convention we will treat is defined by arranging $C_{i j}$ as $^{2}$

$$
C_{i j}=-\psi \delta_{i j}+E_{, i j}+F_{(i, j)}+h_{i j},
$$

in which $\psi$ is the curvature perturbation in this metric convention (as used by Mukhanov, Feldman and Brandenberger in Ref. [18] and Malik and Wands in Ref. [17], for example), $E$ and $F_{i}$ are, respectively, a scalar and a vector part of the spatial metric and $h_{i j}$ is the tensor potential, representing gravitational waves. The vector and tensor quantities above obey

$$
S_{, i}^{i}=0, \quad F_{, i}^{i}=0, \quad h^{i j}{ }_{, j}=0, \quad h^{i}{ }_{i}=0 .
$$

This first convention for $\psi$ can be understood, at first order, as the perturbation to the intrinsic curvature, as explained in Appendix C. The other conventions do not have this property, but can be generally understood as perturbations to the scale factor $a(t)$. Appendix $\mathrm{C}$ also contains a definition of the scale factor from the extrinsic curvature, which is more easily relatable to the versions of $\psi$ given below.

A variation from the form (2.5) consists of collecting the trace of $C_{i j}$ in a single variable, here denoted by $\psi_{T}$. This split was used, e.g., by Bardeen in Ref. [19] and also by Kodama and Sasaki in Ref. [20], where $\psi_{T}$ was denoted by $H_{L}$. The perturbation to the spatial part of the metric becomes

$$
C_{i j}=-\psi_{T} \delta_{i j}+E_{, i j}-\frac{1}{3} \delta_{i j} \nabla^{2} E+F_{(i, j)}+h_{i j},
$$

which, upon comparison with the previous convention, (2.5), shows that the new curvature perturbation $\psi_{T}$ is given by, at all orders

$$
\psi_{T}=\psi-\frac{1}{3} \nabla^{2} E .
$$

\footnotetext{
${ }^{2}$ Note that the definition of the tensor perturbation is slightly different from the one in Ref. [17], i.e. $h_{i j}^{(\mathrm{MW})}=$ $2 h_{i j}$.
} 
The third kind of decomposition of $g_{i j}$ we will treat is similar to the second one, Eq. (2.7), but factors out the determinant of the spatial part of the metric, instead of the trace. This is the decomposition used by Salopek and Bond in Ref. [5] and also by Maldacena in Ref. [21]. It can be written as

$$
g_{i j}=a^{2} e^{2 \psi_{D}}\left[e^{\omega}\right]_{i j},
$$

in which $\omega$ is a traceless tensor and $\psi_{D}$ is the curvature perturbation of interest in this convention, defined by $e^{6 \psi_{D}} \equiv \operatorname{det}\left(g_{i j} / a^{2}\right)$. This quantity is usually interpreted as being a perturbation to the number of e-folds [5], $N$, given by $N=\ln a-\psi_{D}-\psi_{D}^{2}$. A related interpretation would be to think of it as a perturbation to the volume of spatial hypersurfaces, as it is proportional to the determinant of the spatial metric. It can be shown [17], that the first and second order parts of $\psi_{D}$ are related by the following expressions, in the notation of this work

$$
\begin{aligned}
\psi_{D 1}= & -\psi_{T 1}=-\psi_{1}+\frac{1}{3} \nabla^{2} E_{1} \\
\psi_{D 2}= & -\psi_{T 2}-\frac{2}{3} C_{1 i j} C_{1}^{i j}= \\
= & -\psi_{2}+\frac{1}{3} \nabla^{2} E_{2}-2 \psi_{1}^{2}-\frac{2}{3} h_{1 i j} h_{1}^{i j}+\frac{4}{3} \psi_{1} \nabla^{2} E_{1} \\
& -\frac{4}{3} h_{1}^{i j}\left(E_{1, i j}+F_{1 i, j}\right)-\frac{2}{3} F_{1(i, j)} F_{1}^{j, i}-\frac{2}{3} E_{1, i j}\left(2 F_{1}^{i, j}+E_{1}^{, i j}\right) .
\end{aligned}
$$

The fourth convention is not a variation of $g_{i j}$ per se, but only a different way of defining the curvature perturbation. As with the third definition, Eq. (2.9), we factor out the determinant of the spatial part of the metric, but in this case, we use the inverse metric to do so. Therefore, it is now defined as

$$
g^{i j}=a^{-2} e^{-2 \psi_{I}}\left[e^{\omega_{I}}\right]^{i j},
$$

in which, again, $\omega_{I}$ is a traceless tensor and $\psi_{I}$ is the new version of the curvature perturbation, determined by $e^{-6 \psi_{I}} \equiv \operatorname{det}\left(g^{i j} a^{2}\right)$. To our knowledge, this is the first time this definition has been used in the literature. Concerning its interpretation, it can still be seen as a perturbation to the scale factor and we find it to be equal to the integrated expansion, when the latter is evaluated in a comoving threading (see Appendix $\mathrm{C}$ for more details). Comparing this new version of $\psi$ to the original one, we find the following relations

$$
\begin{aligned}
\psi_{I 1}= & -\psi_{1}+\frac{1}{3} \nabla^{2} E_{1}, \\
\psi_{I 2}= & -\psi_{2}+\frac{1}{3} \nabla^{2} E_{2}-2 \psi_{1}^{2}-\frac{2}{3} h_{1 i j} h_{1}^{i j}+\frac{4}{3} \psi_{1} \nabla^{2} E_{1}+\frac{1}{3}\left(B_{1, i}-S_{1 i}\right)\left(B_{1}^{, i}-S_{1}^{i}\right) \\
& -\frac{4}{3} h_{1}^{i j}\left(E_{1, i j}+F_{1 i, j}\right)-\frac{2}{3} F_{1(i, j)} F_{1}^{j, i}-\frac{2}{3} E_{1, i j}\left(2 F_{1}^{i, j}+E_{1}^{, i j}\right) .
\end{aligned}
$$

We will use these four conventions to define different versions of the gauge invariant curvature perturbation in the next section.

\subsection{Stress-energy Tensor}

As for the stress-energy tensor, it is defined in the so-called energy frame $[22,23]^{3}$, including anisotropic stress:

$$
T_{\mu \nu}=(P+\rho) u_{\mu} u_{\nu}+P g_{\mu \nu}+\pi_{\mu \nu}
$$

\footnotetext{
${ }^{3}$ This frame is defined by the condition that the 4 -velocity, $u^{\mu}$, is an eigenvector of $T_{\mu \nu}$, with eigenvalue $\rho$. This is equivalent to setting the energy flux, $q^{\mu}$, to zero.
} 
with

$$
\pi_{\mu}^{\mu}=0, \quad \text { and } \pi_{\mu \nu} u^{\mu}=0 .
$$

The perturbative expansion is the standard one, as given in [17]. We merely reproduce the SVT splitting of $\pi_{2 \mu \nu}$, as it is non-trivial at this order:

$$
\begin{array}{r}
\pi_{200}=0, \quad \pi_{2 i 0}=-2 \pi_{1 i j}\left(v_{V 1}^{j}+v_{1}^{, j}\right), \\
\pi_{2 i j}=a^{2}\left[\Pi_{2 i j}+\Pi_{2(i, j)}+\Pi_{2, i j}-\frac{1}{3} \delta_{i j} \nabla^{2} \Pi_{2}\right]+\frac{4}{3} \delta_{i j} \pi_{1 k l} C_{1}^{k l},
\end{array}
$$

where the quantities $\Pi, \Pi_{i}$ and $\Pi_{i j}$ are, respectivelly, the scalar, vector and tensor parts of the linear piece of the anisotropic stress tensor, $v$ and $v_{V}^{i}$ are the scalar and vector components of the velocity perturbation and $C_{1 i j}$ is the spatial metric perturbation, as given in equation (2.5). The terms that arise in $\pi_{2 i 0}$ and $\pi_{2 i j}$ which depend on the first order anisotropic stress, $\pi_{1 i j}$, are essential in order to not violate the constraints that define the anisotropic stress, Eqs. (2.17). Should these terms not be included, second order contributions of the anisotropic stress to the evolution equations are not well taken into account. That seems to be the case for the energy conservation equation given in [17], which differs from our result presented below in equation (4.4).

\subsection{The gauge problem}

Most of the quantities defined above will depend on a choice of coordinates on the perturbed manifold, the so-called gauge choice [17-20, 24]. A gauge generator vector field $\xi$ is defined to parametrise the change of coordinate system. The gauge transformation it induces in a tensor $T$ is given by

$$
\widetilde{T}=e^{£} T
$$

with $£_{\xi}$ being the Lie derivative in the direction of $\xi$. The transformation rules for the perturbations are obtained by expanding the relation above order by order. Up to second order, the gauge transformations are given by

$$
\begin{array}{r}
\widetilde{\delta T_{1}}=\delta T_{1}+£_{\xi_{1}} T_{0}, \\
\widetilde{\delta T_{2}}=\delta T_{2}+£_{\xi_{2}} T_{0}+£_{\xi_{1}}^{2} T_{0}+2 £_{\xi_{1}} \delta T_{1},
\end{array}
$$

where the gauge generator was also expanded order by order as $\xi^{\mu}=\xi_{1}^{\mu}+\frac{1}{2} \xi_{2}^{\mu}+\ldots$ It can be decomposed further into scalar and vector parts as

$$
\left(\xi^{\mu}\right)=\left(\alpha, \beta^{, i}+\gamma^{i}\right)
$$

Applying these transformations to the metric tensor, one finds the first order metric potentials of the original convention, Eq. (2.5), to change to

$$
\begin{aligned}
& \widetilde{\phi}_{1}=\phi_{1}+\mathcal{H} \alpha_{1}+\alpha_{1}^{\prime}, \quad \widetilde{\psi}_{1}=\psi_{1}-\mathcal{H} \alpha_{1}, \\
& \widetilde{E}_{1}=E_{1}+\beta_{1}, \quad \widetilde{B}_{1}=B_{1}-\alpha_{1}+\beta_{1}^{\prime}, \\
& \widetilde{F}_{1}^{i}=F_{1}^{i}+\gamma_{1}^{i}, \quad \widetilde{S}_{1}^{i}=S_{1}^{i}-\gamma_{1}^{i \prime}, \\
& \widetilde{h}_{1}^{i j}=h_{1}^{i j} \text {, }
\end{aligned}
$$


and the first order fluid quantities to transform to

$$
\begin{array}{ll}
\widetilde{\delta \rho_{1}}=\delta \rho_{1}+\alpha_{1} \rho^{\prime}, & \widetilde{\delta P_{1}}=\delta P_{1}+\alpha_{1} P^{\prime}, \\
\widetilde{v_{1}}=v_{1}-\beta_{1}^{\prime}, & \widetilde{v_{V 1}^{i}}=v_{V 1}^{i}-\gamma_{1}^{i \prime} .
\end{array}
$$

The gauge transformations at second order are given in Appendix A.

The ambiguities created by this gauge issue are solved by "fixing" or choosing a gauge and hence constructing gauge invariant quantities [12, 17-20]. In the following section we derive gauge invariant quantities, specifying a particular gauge by choosing convenient temporal and spatial hypersurfaces, or specifying the slicing and threading, respectively (for a non-technical introduction to the gauge issue in cosmology see Ref. [25]). The gauge invariant quantities are uniquely defined by specifying the hypersurface (at all orders), and can therefore also be evaluated in other gauges, if necessary.

\section{Gauge invariant quantities}

The method we use to generate gauge invariant variables starts with performing a gauge transformation on a variable of interest, e.g. $\psi_{1}$. One then substitutes the gauge generator components $\xi_{1}^{\mu}$ with those obtained by solving a gauge fixing constraint, e.g. $\widetilde{\delta \rho_{1}}=0$. The end result is a gauge invariant quantity, e.g. the curvature perturbation in uniform density hypersurfaces, $\zeta_{1}$. We apply this method for the quantities of interest in the subsections below (for details see e.g. Refs. [17, 24]).

\subsection{Curvature perturbation on uniform density hypersurfaces}

The focus of this work is the curvature perturbation on uniform density hypersurfaces $\zeta$. As was already sketched above, it is defined to be equal to $-\psi$ in the gauge in which the density field is uniform $(\widetilde{\delta \rho}=0)$. Starting with our first convention for the metric, Eq. (2.5), this condition is sufficient to fully construct $\zeta$ at first order as $([4,8])$

$$
\zeta_{1} \equiv-\psi_{1}-\mathcal{H} \frac{\delta \rho_{1}}{\rho^{\prime}} .
$$

However, at second order, one is also forced to specify the first order gauge to define this curvature perturbation unambiguously. For this convention of the metric tensor, Eq. (2.5), we will use the following gauge conditions to define $\zeta_{2}([12])$

$$
\zeta_{2} \equiv-\widetilde{\psi_{2}}, \text { if } \widetilde{\delta \rho_{2}}=\widetilde{\delta \rho_{1}}=\widetilde{E_{1}}=0, \widetilde{F_{1}^{i}}=0 .
$$

These add a flat threading to the uniform density gauge. The general formal expression for $\zeta_{2}$ is given in [17]. In full detail, the formula is rather complicated and we write it here with the r.h.s. evaluated in flat gauge ${ }^{4}$,

$$
\begin{aligned}
\zeta_{2}= & -\frac{\mathcal{H}}{\rho^{\prime}} \delta \rho_{2}+\frac{1}{\rho^{\prime 2}}\left(2 \mathcal{H}^{2}+\mathcal{H}^{\prime}-\mathcal{H} \frac{\rho^{\prime \prime}}{\rho^{\prime}}\right) \delta \rho_{1}^{2}+\frac{2 \mathcal{H}}{\rho^{\prime 2}} \delta \rho_{1} \delta \rho_{1}^{\prime}-\frac{1}{2 \rho^{\prime 2}} \delta \rho_{1, k} \delta \rho_{1}^{, k}-\frac{1}{\rho^{\prime}}\left(B_{1, k}-S_{1 k}\right) \delta \rho_{1}^{, k} \\
& +\nabla^{-2}\left\{\frac{1}{2}\left[\frac{1}{\rho^{\prime 2}} \delta \rho_{1}^{, i} \delta \rho_{1}^{, j}+\frac{2}{\rho^{\prime}}\left(\delta \rho_{1}^{,(i} B_{1}^{, j)}-\delta \rho_{1}^{,(i} S_{1}^{j)}\right)\right]_{, i j}+\frac{1}{\rho^{\prime}}\left(h_{1}^{i j \prime}+2 \mathcal{H} h_{1}^{i j}\right) \delta \rho_{1, i j}\right\} .
\end{aligned}
$$

\footnotetext{
${ }^{4}$ Flat gauge is defined by the conditions $\psi=E=F^{i}=0$.
} 
We can see that, in contrast with the first order result, the second order $\zeta$ is much harder to relate to density perturbations in flat gauge, given the presence of vectors and tensors. In spite of this, this expression is still useful in writing the gauge invariant curvature perturbation in terms of multiple scalar fields, as is done in [26, 27].

Let us now move to the second convention of the metric, Eq. (2.7). In this case, $\widetilde{\delta \rho}=0$ is no longer a sufficient gauge condition to define an invariant, even at first order; one must also specify the scalar part of the threading, due to the inclusion of $E$ in the definition of $\psi_{T}$ (see Eq. (2.8)). The extra condition that is most often chosen is $\widetilde{v_{1}}=0$, which results in the following expression ${ }^{5}$

$$
\zeta_{T 1}=-\psi_{T 1}-\frac{\mathcal{H}}{\rho^{\prime}} \delta \rho_{1}+\frac{1}{3} \nabla^{2} \int v_{1} d \tau,
$$

in which the integral in conformal time is indefinite. The introduction of these integrals is the disadvantage of using the gauge condition, $\widetilde{v_{1}}=0$. This might be problematic, as this condition only sets the gauge up to an arbitrary function of the spatial coordinates, which, in turn, might spoil the gauge invariance of the new variable. In spite of this, it is possible to construct a gauge invariant quantity, by defining it to be

$$
\zeta_{T 1} \equiv \zeta_{1}+\frac{1}{3} \nabla^{2} \int J_{1} d \tau
$$

with $J_{1}$ being the gauge invariant velocity on flat hypersurfaces, defined by

$$
J_{1}=E_{1}^{\prime}+v_{1} .
$$

While the integral in Eq. (3.5) is still indefinite, the integrand is gauge invariant and, therefore, this is the definition we use.

At second order, one sets the second order gauge in the same way, i.e. $\widetilde{\delta \rho_{2}}=\widetilde{v_{2}}=0$ and, to avoid additional issues with indefinite integrals, one can choose $\widetilde{\delta \rho_{1}}=\widetilde{E_{1}}=\widetilde{F_{1}^{i}}=0$ for the first order gauge fixing. With this choice, we find

$$
\zeta_{T 2}=\zeta_{2}+\frac{1}{3} \nabla^{2} \int J_{2} d \tau
$$

in which $J_{2}$ is the second order equivalent of $J_{1}$ in this gauge, i.e. it equals $E_{2}^{\prime}+v_{2}$ in the gauge obeying $\widetilde{\delta \rho_{1}}=\widetilde{E_{1}}=\widetilde{F_{1}^{i}}=0$. As is visible in the expression above, Eq. (3.5), the only variable of interest is $\nabla^{2} J_{2}$ and hence, for shortness of presentation, that is all we show below, with the r.h.s. evaluated in flat gauge

$$
\begin{aligned}
\nabla^{2} J_{2}= & \nabla^{2} v_{2}+\frac{2}{\rho^{\prime}}\left[\delta \rho_{1}\left(\mathcal{H}\left(v_{V 1}^{i}+v_{1}^{, i}\right)-v_{V 1}^{i \prime}-v_{1}^{\prime, i}\right)\right]_{, i}+\left[\frac{\delta \rho_{1, i} \delta \rho_{1}^{, i}}{2 \rho^{\prime 2}}+\frac{\left(B_{1}^{, i}-S_{1}^{i}\right) \delta \rho_{1, i}}{\rho^{\prime}}\right. \text { (3.8) } \\
& \left.+\nabla^{-2}\left\{-\frac{3}{2}\left[\frac{1}{\rho^{\prime 2}} \delta \rho_{1}^{, i} \delta \rho_{1}^{, j}+\frac{2}{\rho^{\prime}}\left(\delta \rho_{1}^{,(i} B_{1}^{, j)}-\delta \rho_{1}^{,(i} S_{1}^{j)}\right)\right]_{, i j}-\frac{3}{\rho^{\prime}}\left(h_{1}^{i j \prime}+2 \mathcal{H} h_{1}^{i j}\right) \delta \rho_{1, i j}\right\}\right]^{\prime} .
\end{aligned}
$$

As we will see in Section 4, this quantity is relevant regardless of the choice of convention for the metric, as it will appear in the evolution equation for the curvature perturbation.

\footnotetext{
${ }^{5}$ An alternative choice would be $\widetilde{E_{1}}=0$, but that would simply result in the expression for the original metric convention, as $\widetilde{\psi_{T 1}}=\widetilde{\psi_{1}}$.
} 
Let us now turn to the third convention of the metric, Eq. (2.9). For this case, $\zeta_{D}$ will be defined as being equal to $\psi_{D}$ instead of $-\psi_{D}$, in order to keep the same sign as $\zeta$. Starting at first order, we see that we get either $\zeta_{D 1}=\zeta_{1}$ or $\zeta_{D 1}^{(v)}=\zeta_{T 1}$, depending on whether we choose $\widetilde{E_{1}}=0$ or $\widetilde{v_{1}}=0$, respectively, for fixing the threading. The second order result is more interesting, as there is no gauge fixing for which it is equal to either of the other definitions above. In the most conservative case, the choice of gauge fixing is $\widetilde{\delta \rho_{2}}=\widetilde{E_{2}}=0$ at second order and $\widetilde{\delta \rho_{1}}=\widetilde{E_{1}}=\widetilde{F_{1}^{i}}=0$ at first order. This results in 6

$$
\zeta_{D 2}=\zeta_{2}-\frac{2}{3} h_{1 i j} h_{1}^{i j}-2 \zeta_{1}^{2}
$$

A different gauge fixing is $\widetilde{\delta \rho_{2}}=\widetilde{v_{2}}=0$ and $\widetilde{\delta \rho_{1}}=\widetilde{v_{1}}={\widetilde{v_{V 1}}}^{i}=0$, for which the result is

$$
\begin{aligned}
\zeta_{D 2}^{(v)}= & \zeta_{2}+\frac{1}{3} \int \nabla^{2} J_{2} d \tau-\frac{2}{3} h_{1 i j} h_{1}^{i j}-2 \zeta_{1}^{2} \\
& +2 \zeta_{1, i} \int\left(J_{1}^{i}+V_{1}^{i}\right) d \tau+\frac{2}{3} \int\left\{\left[\left(J_{1}^{i}+V_{1}^{i}\right) \Upsilon_{1}\right]_{, i}+\nabla^{2} J_{1, i} \int\left(J_{1}^{i}+V_{1}^{i}\right) d \tau^{\prime}\right\} d \tau
\end{aligned}
$$

in which $V_{1}^{i}$ is the gauge invariant velocity vector perturbation in flat hypersurfaces and $\Upsilon$ is the gauge invariant lapse perturbation in uniform density hypersurfaces. In a general gauge, these quantities are given by

$$
\begin{aligned}
& V_{1}^{i}=v_{V 1}^{i}+F_{1}^{i \prime}, \\
& \Upsilon_{1}=\phi_{1}-\mathcal{H} \frac{\delta \rho_{1}}{\rho^{\prime}}-\left(\frac{\delta \rho_{1}}{\rho^{\prime}}\right)^{\prime} .
\end{aligned}
$$

For the forth version of the curvature perturbation, Eq. (2.13), the procedure is very similar to the one for the third convention. As in the previous case, the first order quantities obey $\zeta_{I 1}=\zeta_{1}$ or $\zeta_{I 1}^{(v)}=\zeta_{T 1}$, depending on whether $\widetilde{E_{1}}=0$ or $\widetilde{v_{1}}=0$ is chosen for setting the threading. At second order, the results are

$$
\zeta_{I 2}=\zeta_{2}-\frac{2}{3} h_{1 i j} h_{1}^{i j}-2 \zeta_{1}^{2}+\frac{1}{3}\left(W_{1 i}-V_{1 i}+A_{1, i}-J_{1, i}\right)\left(W_{1}^{i}-V_{1}^{i}+A_{1}^{, i}-J_{1}^{, i}\right),
$$

if the gauge is fixed with $\widetilde{\delta \rho_{2}}=\widetilde{E_{2}}=0$ and $\widetilde{\delta \rho_{1}}=\widetilde{E_{1}}=\widetilde{F_{1}^{i}}=0$, and

$$
\begin{aligned}
\zeta_{I 2}^{(v)}= & \zeta_{2}+\frac{1}{3} \int \nabla^{2} J_{2} d \tau-\frac{2}{3} h_{1 i j} h_{1}^{i j}-2 \zeta_{1}^{2}+\frac{1}{3}\left(W_{1 i}+A_{1, i}\right)\left(W_{1}^{i}+A_{1}^{, i}\right) \\
& +2 \zeta_{1, i} \int\left(J_{1}^{i}+V_{1}^{i}\right) d \tau+\frac{2}{3} \int\left\{\left[\left(J_{1}^{i}+V_{1}^{i}\right) \Upsilon_{1}\right]_{, i}+\nabla^{2} J_{1, i} \int\left(J_{1}^{\prime i}+V_{1}^{i}\right) d \tau^{\prime}\right\} d \tau
\end{aligned}
$$

when the gauge choice is $\widetilde{\delta \rho_{2}}=\widetilde{v_{2}}=0$ and $\widetilde{\delta \rho_{1}}=\widetilde{v_{1}}={\widetilde{v_{V 1}}}^{i}=0$. The new first order gauge invariant quantities that appear are the vector velocity in zero shift gauge, $W_{1}^{i}$, and the momentum perturbation in uniform density gauge, $A_{1}$. They are given by

$$
\begin{aligned}
& W_{1}^{i}=v_{V 1}^{i}-S_{1}^{i}, \\
& A_{1}=v_{1}+B_{1}+\frac{\delta \rho_{1}}{\rho^{\prime}} .
\end{aligned}
$$

\footnotetext{
${ }^{6}$ This result is well known in the case without tensors. See, for example, Refs. [7, 11, 27].
} 


\section{$3.2 \quad$ Non-adiabatic pressure}

One of the quantities determining the evolution of the curvature perturbation is the nonadiabatic pressure $[8,19,20,28,29]$. It is defined as the deviation from the adiabatic relation as

$$
\delta P=c_{\mathrm{s}}^{2} \delta \rho+\delta P_{\mathrm{nad}},
$$

with $c_{\mathrm{s}}$ the adiabatic sound speed defined as $c_{\mathrm{s}}^{2}=P^{\prime} / \rho^{\prime}$. At first order, this definition automatically generates a gauge invariant quantity, but, at second order, this is not sufficient and many choices can be made. Our first choice is to define it as the gauge invariant quantity that reduces to Eq. (3.17) in the gauge in which $\widetilde{\delta \rho_{1}}=\widetilde{E_{1}}=\widetilde{F_{1}^{i}}=0$. In a general gauge, this quantity is given by

$$
\begin{aligned}
\delta P_{\text {nad } 2}= & \delta P_{2}-c_{\mathrm{s}}^{2} \delta \rho_{2}-\frac{2}{\rho^{\prime}} \delta \rho_{1} \delta P_{1}^{\prime}+\left(\frac{P^{\prime \prime}}{\rho^{\prime 2}}-\frac{P^{\prime} \rho^{\prime \prime}}{\rho^{\prime 3}}\right) \delta \rho_{1}^{2} \\
& +\frac{2 c_{s}^{2}}{\rho^{\prime}} \delta \rho_{1} \delta \rho_{1}^{\prime}-2\left(F_{1}^{i}+E_{1}^{, i}\right) \delta P_{\text {nad } 1, i} .
\end{aligned}
$$

With the different choice of threading, $\widetilde{v_{1}}={\widetilde{v_{V 1}}}^{i}=0$, one finds instead

$$
\delta P_{\text {nad } 2}^{(v)}=\delta P_{\text {nad } 2}+2 \delta P_{\text {nad } 1, i} \int\left(V_{1}^{i}+J_{1}^{, i}\right) d \tau .
$$

For a barotropic fluid, with $P=P(\rho)$, both expressions vanish, as can be easily checked by evaluating them in their defining gauge, i.e. with $\delta \rho_{1}=E_{1}=F_{1}^{i}=0$.

The quantities presented so far include the full set of gauge invariant quantities required for the full derivation of the evolution equations below.

\section{Evolution equations}

In this section, we present the derivation of the evolution equations for all versions of $\zeta$. Our strategy consists of calculating the derivative of expression (3.3) and using only the perturbed energy-momentum conservation equations up to second order to simplify the result. Lastly, we substitute the gauge dependent variables for gauge invariant ones, using the expressions found in the previous Section, to arrive at our final result. Having found the result for $\zeta_{2}$ in the original convention of the metric, Eq. (2.5), we then rewrite the evolution equation in terms of the different definitions of $\zeta$.

\subsection{Fluid equations}

Energy-momentum conservation, $T_{; \nu}^{\mu \nu}=0$, governs the evolution of the fluid density and velocity. For simplicity, we present these evolution equations in flat gauge. The first order energy conservation equation is given by

$$
\delta \rho_{1}^{\prime}+3 \mathcal{H}\left(\delta \rho_{1}+\delta P_{1}\right)+(\rho+P) \nabla^{2} v_{1}=0
$$

while momentum conservation is

$$
\delta P_{1, k}+(\rho+P)\left[Z_{1 k}^{\prime}+\phi_{1, k}+\left(1-3 c_{s}^{2}\right) \mathcal{H} Z_{1 k}\right]+\frac{2}{3} \nabla^{2} \Pi_{1, k}+\frac{1}{2} \nabla^{2} \Pi_{1 k}=0,
$$


where the momentum perturbation $Z_{1}{ }^{k}$ is given by

$$
Z_{1}{ }^{k}=v_{V 1}^{k}-S_{1}{ }^{k}+B_{1}{ }^{k}+v_{1}{ }^{, k}
$$

At second order, we only require the energy conservation equation, which is

$$
\begin{aligned}
\delta \rho_{2}^{\prime}= & -3 \mathcal{H}\left(\delta \rho_{2}+\delta P_{2}\right)-(\rho+P) \nabla^{2} v_{2}-2\left(\delta P_{1}+\delta \rho_{1}\right) \nabla^{2} v_{1}-2 \delta \rho_{1, k}\left(v_{V 1}^{k}+v_{1}^{, k}\right) \\
& -2 \delta P_{1, k} Z_{1}{ }^{k}-(\rho+P)\left[4 Z_{1 k}^{\prime} Z_{1}{ }^{k}+2\left(1-3 c_{s}^{2}\right) \mathcal{H} Z_{1 k} Z_{1}{ }^{k}+2 \phi_{1, k} Z_{1}{ }^{k}\right. \\
& \left.+2 \phi_{1, k}\left(v_{V 1}^{k}+v_{1}{ }^{k}\right)+2 \phi_{1} \nabla^{2} v_{1}-4 h_{1 i j}^{\prime} h_{1}^{i j}\right]-Z_{1}{ }^{k}\left(\frac{4}{3} \nabla^{2} \Pi_{1, k}+\nabla^{2} \Pi_{1 k}\right) \\
& -2\left(h_{1 i j}^{\prime}+v_{V 1 i, j}+v_{1, i j}\right)\left(\Pi_{1}^{i j}+\Pi_{1}{ }^{(i, j)}+\Pi_{1}{ }^{i j}-\frac{1}{3} \delta^{i j} \nabla^{2} \Pi_{1}\right) .
\end{aligned}
$$

The above equations are sufficient to derive evolution equations for the curvature perturbation at first and at second order [8].

\subsection{Evolution of the curvature perturbation}

We can now derive the evolution equation for the curvature perturbation on uniform density hypersurfaces. We follow the strategy stated at the beginning of this section. At first order, the result is well known to be

$$
\zeta_{1}^{\prime}=-\frac{1}{3} \nabla^{2} J_{1}-\mathcal{H} \frac{\delta P_{\text {nad } 1}}{\rho+P},
$$

where only the first order energy conservation equation was used. On large scales (" $\nabla \rightarrow 0$ ") and in the absence of non-adiabatic pressure, one finds the familiar conservation equation $\zeta_{1}^{\prime}=0$.

For the other conventions for the curvature perturbation, $\zeta_{T 1}, \zeta_{D 1}^{(v)}$ and $\zeta_{I 1}^{(v)}$, the evolution equation at first order is the same and is given by

$$
\zeta_{T 1}^{\prime}=-\mathcal{H} \frac{\delta P_{\operatorname{nad} 1}}{\rho+P}
$$

which shows these versions of $\zeta_{1}$ are conserved at all scales, when non-adiabatic pressure is negligible $[9,30]$.

At second order, the complexity increases. The procedure to obtain the final result is as follows: use the energy conservation equation at first (Eq. (4.1)) and second order (Eq. (4.4)) to substitute for $\delta \rho_{1}^{\prime}$ and $\delta \rho_{2}^{\prime}$ and substitute $\left(\frac{4}{3} \nabla^{2} \Pi_{1, k}+\nabla^{2} \Pi_{1 k}\right)$ with the momentum conservation equation, Eq. (4.2). The last step is to use the defining expressions of the gauge invariants to eliminate all gauge dependant variables. The final result is given by ${ }^{7}$

$$
\begin{aligned}
& \left(-\zeta_{2}+2 \zeta_{1}^{2}-\frac{1}{3}\left(W_{1 i}+A_{1, i}\right)\left(W_{1}^{i}+A_{1}^{, i}\right)+\frac{2}{3} h_{1 i j} h_{1}^{i j}\right)^{\prime}= \\
& \frac{1}{3} \nabla^{2} J_{2}+\mathcal{H} \frac{\delta P_{\mathrm{nad} 2}}{\rho+P}-2 \mathcal{H} \frac{\delta P_{\mathrm{nad} 1}^{2}}{(\rho+P)^{2}}+\frac{2}{3}\left[\Upsilon\left(V_{1}^{i}+J_{1}^{, i}\right)\right]_{, i}+2 \zeta_{1, i}\left(V_{1}^{i}+J_{1}^{, i}\right) \\
& -\frac{2 \mathcal{H}}{\rho^{\prime}}\left(\Pi_{1 i j}+\Pi_{1(i, j)}+\Pi_{1, i j}-\frac{1}{3} \delta_{i j} \nabla^{2} \Pi_{1}\right)\left(h_{1}^{i j \prime}+V_{1}^{i, j}+J_{1}^{, i j}\right) .
\end{aligned}
$$

\footnotetext{
${ }^{7}$ Note the absence of inverse Laplacians. That is explained by an exact cancellation between the terms in $\zeta_{2}^{\prime}$ and those in $\nabla^{2} J_{2}$, as can be shown by comparing equations (3.8) and (3.3).
} 
We are now able to identify the different terms that source the evolution of $\zeta_{2}$. We note, in particular, the appearance of vector and tensor source terms as well as the anisotropic stress which did not appear at first order in this equation ${ }^{8}$.

We are now in the position to substitute for the other versions of $\zeta$ and find their evolution equations. For $\zeta_{2 T}$, we find

$$
\begin{aligned}
& \left(-\zeta_{2 T}+2 \zeta_{1}^{2}-\frac{1}{3}\left(W_{1 i}+A_{1, i}\right)\left(W_{1}^{i}+A_{1}^{, i}\right)+\frac{2}{3} h_{1 i j} h_{1}^{i j}\right)^{\prime}= \\
& \mathcal{H} \frac{\delta P_{\text {nad } 2}}{\rho+P}-2 \mathcal{H} \frac{\delta P_{\text {nad } 1}^{2}}{(\rho+P)^{2}}+\frac{2}{3}\left[\Upsilon\left(V_{1}^{i}+J_{1}^{, i}\right)\right]_{, i}+2 \zeta_{1, i}\left(V_{1}^{i}+J_{1}^{, i}\right) \\
& -\frac{2 \mathcal{H}}{\rho^{\prime}}\left(\Pi_{1 i j}+\Pi_{1(i, j)}+\Pi_{1, i j}-\frac{1}{3} \delta_{i j} \nabla^{2} \Pi_{1}\right)\left(h_{1}^{i j \prime}+V_{1}^{i, j}+J_{1}^{, i j}\right),
\end{aligned}
$$

while $\zeta_{D 2}$ evolves as

$$
\begin{aligned}
& \left(-\zeta_{D 2}-\frac{1}{3}\left(W_{1 i}+A_{1, i}\right)\left(W_{1}^{i}+A_{1}^{i}\right)\right)^{\prime}= \\
& \frac{1}{3} \nabla^{2} J_{2}+\mathcal{H} \frac{\delta P_{\mathrm{nad} 2}}{\rho+P}-2 \mathcal{H} \frac{\delta P_{\mathrm{nad} 1}^{2}}{(\rho+P)^{2}}+\frac{2}{3}\left[\Upsilon\left(V_{1}^{i}+J_{1}^{, i}\right)\right]_{, i}+2 \zeta_{D 1, i}\left(V_{1}^{i}+J_{1}^{i}\right) \\
& -\frac{2 \mathcal{H}}{\rho^{\prime}}\left(\Pi_{1 i j}+\Pi_{1(i, j)}+\Pi_{1, i j}-\frac{1}{3} \delta_{i j} \nabla^{2} \Pi_{1}\right)\left(h_{1}^{i j \prime}+V_{1}^{i, j}+J_{1}^{, i j}\right),
\end{aligned}
$$

and the result for $\zeta_{D 2}^{(v)}$ is

$$
\begin{aligned}
& \left(-\zeta_{D 2}^{(v)}-\frac{1}{3}\left(W_{1 i}+A_{1, i}\right)\left(W_{1}^{i}+A_{1}^{, i}\right)\right)^{\prime}=\mathcal{H} \frac{\delta P_{\text {nad } 2}^{(v)}}{\rho+P}-2 \mathcal{H} \frac{\delta P_{\text {nad } 1}^{2}}{(\rho+P)^{2}} \\
& -\frac{2 \mathcal{H}}{\rho^{\prime}}\left(\Pi_{1 i j}+\Pi_{1(i, j)}+\Pi_{1, i j}-\frac{1}{3} \delta_{i j} \nabla^{2} \Pi_{1}\right)\left(h_{1}^{i j \prime}+V_{1}^{i, j}+J_{1}^{, i j}\right) .
\end{aligned}
$$

The simplest evolutions equations are found for the $\zeta_{I 2}$ and $\zeta_{I 2}^{(v)}$ versions of the gauge invariant curvature perturbation. They are given by

$$
\begin{aligned}
& \left(-\zeta_{I 2}+\frac{1}{3}\left(V_{1 i}+J_{1, i}\right)\left(V_{1}^{i}+J_{1}^{, i}-2 W_{1}^{i}-2 A_{1}^{, i}\right)\right)^{\prime}= \\
& \frac{1}{3} \nabla^{2} J_{2}+\mathcal{H} \frac{\delta P_{\text {nad } 2}}{\rho+P}-2 \mathcal{H} \frac{\delta P_{\text {nad } 1}^{2}}{(\rho+P)^{2}}+\frac{2}{3}\left[\Upsilon\left(V_{1}^{i}+J_{1}^{, i}\right)\right]_{, i}+2 \zeta_{I 1, i}\left(V_{1}^{i}+J_{1}^{i}\right) \\
& -\frac{2 \mathcal{H}}{\rho^{\prime}}\left(\Pi_{1 i j}+\Pi_{1(i, j)}+\Pi_{1, i j}-\frac{1}{3} \delta_{i j} \nabla^{2} \Pi_{1}\right)\left(h_{1}^{i j \prime}+V_{1}^{i, j}+J_{1}^{, i j}\right),
\end{aligned}
$$

\footnotetext{
${ }^{8}$ Note however, that the scalar part of the anisotropic stress tensor would source the evolution of $\zeta$ at first order by acting on the evolution of $\nabla^{2} J$. This can be seen more clearly by deriving Eq. (4.5) and using the momentum conservation equation, Eq. (4.2), to substitute for $\nabla^{2} J$ :

$$
\zeta_{1}^{\prime \prime}+\mathcal{H} \zeta_{1}^{\prime}-\frac{P^{\prime}}{3(\rho+P)} \nabla^{2} A_{1}-\frac{1}{3} \nabla^{2} \Phi_{1}+\left(\mathcal{H} \frac{\delta P_{\mathrm{nad} 1}}{\rho+P}\right)^{\prime}+\mathcal{H}^{2} \frac{\delta P_{\mathrm{nad} 1}}{\rho+P}-\frac{\nabla^{2} \delta P_{\mathrm{nad} 1}}{3(\rho+P)}-\frac{2}{9(\rho+P)} \nabla^{2} \nabla^{2} \Pi_{1}=0,
$$

in which $\Phi_{1}$ is one of the Bardeen potentials, given in terms of our variables as $\Phi_{1}=\Upsilon_{1}+\mathcal{H}\left(A_{1}-J_{1}\right)+\left(A_{1}-J_{1}\right)^{\prime}$. 
and

$$
\begin{aligned}
& -\zeta_{I 2}^{(v) \prime}=\mathcal{H} \frac{\delta P_{\text {nad } 2}^{(v)}}{\rho+P}-2 \mathcal{H} \frac{\delta P_{\text {nad } 1}^{2}}{(\rho+P)^{2}} \\
& -\frac{2 \mathcal{H}}{\rho^{\prime}}\left(\Pi_{1 i j}+\Pi_{1(i, j)}+\Pi_{1, i j}-\frac{1}{3} \delta_{i j} \nabla^{2} \Pi_{1}\right)\left(h_{1}^{i j \prime}+V_{1}^{i, j}+J_{1}^{i j}\right) .
\end{aligned}
$$

This final expression, like its first order version, Eq. (4.6), shows that, in the absence of non-adiabatic pressure and anisotropic stress, this version of the curvature perturbation is conserved on all scales. While this is interesting, in order for this result to be useful, one would likely be forced to estimate the integrals in the defining expression for $\zeta_{I 2}$, Eq. (3.14). This is not likely to be straightforward, given the indeterminate nature of the integrals. This evolution equation matches the results of Ref. [31] for the integrated expansion in the absence of anisotropic stress, obtained in the covariant approach.

\subsection{Large scale approximation}

Here we perform the large scale approximation, by neglecting all terms with spatial derivatives in the equations above ${ }^{9}$. This limit simplifies the evolution equations to

$$
\left(-\zeta_{2}+2 \zeta_{1}^{2}-\frac{1}{3} W_{1 i} W_{1}^{i}+\frac{2}{3} h_{1 i j} h_{1}^{i j}\right)^{\prime}=\mathcal{H} \frac{\delta P_{\operatorname{nad} 2}}{\rho+P}-2 \mathcal{H} \frac{\delta P_{\text {nad } 1}^{2}}{(\rho+P)^{2}}-\frac{2 \mathcal{H}}{\rho^{\prime}} \Pi_{1 i j} h_{1}^{i j \prime},
$$

for $\zeta_{2}$, here representing both the original $\zeta_{2}$ and $\zeta_{T 2}$, and

$$
\left(-\zeta_{D 2}-\frac{1}{3} W_{1 i} W_{1}^{i}\right)^{\prime}=\mathcal{H} \frac{\delta P_{\text {nad } 2}}{\rho+P}-2 \mathcal{H} \frac{\delta P_{\text {nad } 1}^{2}}{(\rho+P)^{2}}-\frac{2 \mathcal{H}}{\rho^{\prime}} \Pi_{1 i j} h_{1}^{i j \prime},
$$

for the evolution of both $\zeta_{D 2}$ and $\zeta_{D 2}^{(v)}$, and

$$
\begin{aligned}
\left(-\zeta_{I 2}+\frac{1}{3} V_{1 i}\left(V_{1}^{i}-2 W_{1}^{i}\right)\right)^{\prime} & =\mathcal{H} \frac{\delta P_{\mathrm{nad} 2}}{\rho+P}-2 \mathcal{H} \frac{\delta P_{\mathrm{nad} 1}^{2}}{(\rho+P)^{2}}-\frac{2 \mathcal{H}}{\rho^{\prime}} \Pi_{1 i j} h_{1}^{i j \prime}, \\
-\zeta_{I 2}^{(v) \prime} & =\mathcal{H} \frac{\delta P_{\mathrm{nad} 2}}{\rho+P}-2 \mathcal{H} \frac{\delta P_{\mathrm{nad} 1}^{2}}{(\rho+P)^{2}}-\frac{2 \mathcal{H}}{\rho^{\prime}} \Pi_{1 i j} h_{1}^{i j \prime} .
\end{aligned}
$$

Note that, in all cases above, the pairs are equal in the large scale approximation, except for $\zeta_{I 2}$ and $\zeta_{I 2}^{(v)}$, which have a different contribution from vector perturbations. We give the expressions for each version in this approximation in Appendix B. From this result, one can see that, even in the absence of the scalar non-adiabatic pressure, $\delta P_{\text {nad }}$, neither curvature perturbation is conserved,

$$
\left(-\zeta_{2}+\frac{2}{3} h_{1 i j} h_{1}^{i j}-\frac{1}{3} W_{1}^{i} W_{1 i}\right)^{\prime}=\left(-\zeta_{D 2}-\frac{1}{3} W_{1}^{i} W_{1 i}\right)^{\prime}=-\zeta_{I 2}^{(v) \prime}=-\frac{2 \mathcal{H}}{\rho^{\prime}} \Pi_{1 i j} h_{1}^{i j \prime} .
$$

However, if the traceless, transverse part of the anisotropic stress, $\Pi_{1 i j}$, is negligible, $\zeta_{I 2}^{(v)}$ is in fact conserved

$$
\zeta_{I 2}^{(v) \prime}=\left(\zeta_{D 2}+\frac{1}{3} W_{1}^{i} W_{1 i}\right)^{\prime}=\left(\zeta_{2}+\frac{1}{3} W_{1}^{i} W_{1 i}-\frac{2}{3} h_{1}^{i j} h_{1 i j}\right)^{\prime}=0 .
$$

\footnotetext{
${ }^{9}$ This is generally well motivated in the case of some metric potentials, as one expects the perturbed metric to approach the background metric on large scales [9], and we will assume the same is true for the matter variables, including the anisotropic stress. Should this assumption not hold for the particular model under study, then the results in this section are not valid and one should use the full results from section 4.2.
} 
Although $\zeta_{I 2}^{(v)}$ is exactly conserved, the difference between $\zeta_{I 2}^{(v) \prime}$ and $\zeta_{D 2}^{\prime}$ only depends on vector perturbations, which are usually negligible. Moreover, using the vector part of the momentum conservation equation, Eq. (4.2), in the absence of anisotropic stress, we find the evolution of $W_{1}^{i}$ is given by

$$
W_{1 i}^{\prime}+\mathcal{H}\left(1-3 c_{s}^{2}\right) W_{1 i}=0
$$

Thus, this vector perturbation is conserved during radiation domination $\left(c_{s}^{2}=1 / 3\right)$ and, as a consequence, $\zeta_{D 2}$ is exactly conserved during that epoch. In the general case, we may therefore write the evolution of $\zeta_{D 2}$ on large scales as

$$
\zeta_{D 2}^{\prime}=-\frac{2}{3} \mathcal{H}\left(1-3 c_{s}^{2}\right) W_{1}^{i} W_{1 i}
$$

showing again that it may only have an appreciable evolution if the vector modes are large.

The evolution equations simplify further in Einstein gravity, as, in the absence of anisotropic stress, tensor modes stop evolving and hence this new conservation law converges fairly quickly to the conservation of $\zeta_{2}$ itself. Hence, for Einstein gravity, all versions of the curvature perturbation are conserved up to second order on large scales, if both the non-adiabatic pressure and the anisotropic stress are negligible. However, should the evolution of vectors and tensors be appreciable, the version of $\zeta$ which is conserved is $\zeta_{I}^{(v)}$, i.e., the version defined by the determinant of $g^{i j}$ and by using a comoving threading to fix the gauge.

\section{Conclusion}

We obtained the evolution equation for the curvature perturbation at second order in cosmological perturbation theory, valid on all scales. With the inclusion of vectors, tensors and anisotropic stress, this result allows for high precision calculations of correlation functions on all scales. We derive this for six different definitions of $\zeta$, based on several different splits of the spatial metric and on various choices of the defining gauge. The results for the evolution equations show a substantial difference in apparent complexity, being simpler when the threading defining $\zeta$ was chosen to be the comoving one, i.e. $\widetilde{v}^{i}=0$. Eq. (4.13) for the evolution of $\zeta_{I 2}^{(v)}$ is particularly short, but its usefulness is unclear due to the existence of indefinite time integrals in the definitions of $\zeta_{I 2}^{(v)}$ and $\delta P_{\text {nad } 2}^{(v)}$. On the other hand, for the versions of $\zeta$ for which the threading was chosen with $\widetilde{E}=0$, or the original $\zeta_{2}$, the definitions include inverse Laplacians (see Eq. (3.3)). In both cases, non-locality is present in some form, either in time or in space, and there is no version of the curvature perturbation which evades both of these issues. However, in both cases, the difficulties of the calculation are resolved by solving additional differential equations, both of which require boundary conditions. In the case of the inverse Laplacian, the equation to solve is a Poisson equation, which only depends on first order quantities at a single time, while for the case of the integrals in time, knowledge of the full time evolution of second order quantities is required $\left(\nabla^{2} J_{2}\right.$ in Eq. (3.14), for example). This seems to render the quantities without integrals in time more amenable for situations that require the calculation of $\zeta$ from its definition, such as when its value is evaluated from the value of scalar field or density perturbations. In any case, all these issues disappear in the large scale approximation, for which the inverse Laplacian term in question has a well defined limit and the integrals vanish.

Moreover, we found that, on large scales, the evolution of $\zeta$ is sourced by the transverse traceless part of the anisotropic stress tensor, as well as non-adiabatic pressure. Both 
quantities must therefore be negligible for any version of $\zeta$ to be conserved. Furthermore, the version of the curvature perturbation which is exactly conserved is the one based on the determinant of $g^{i j}$ and comoving threading, $\zeta_{I}^{(v)}$, Eq. (3.14). Other definitions may evolve with the evolution of tensor and vector modes, should such an evolution be allowed by the theory of gravitation under study. For General Relativity, however, vector perturbations are usually very small and the evolution of tensor modes is negligible in the absence of anisotropic stress; therefore all versions of the curvature perturbation are approximately conserved on large scales.

The results presented here are valid as long as the energy and momentum conservation equations, Eqs. (4.1), (4.2) and (4.4), are satisfied. This will be true if the stress-energy tensor is covariantly conserved, i.e. $\nabla_{\mu} T^{\mu \nu}=0$, and the connection is the Levi-Civita connection (i.e. no torsion is present). This is the case in GR, but also in other theories, such as Massive Gravity and Bigravity [32, 33]. The latter theories are interesting in this context, as the tensor modes evolve differently due to the non-zero mass of the graviton [34] and therefore, $\zeta_{D}$ and $\zeta_{I}$ would be the only versions of the curvature perturbation that are conserved.

Furthermore, the usefulness of these results may be extended to theories of gravity for which $\nabla_{\mu} T^{\mu \nu} \neq 0$. This is possible if one can perform a conformal transformation to the Einstein frame and apply the same ideas to the effective stress-energy tensor that arises as the r.h.s. of the new field equations. The difference between our standard scenario and a modified one is that the effective matter quantities thus defined, would not have the same physical significance as the ones we use in this work. Therefore, in those modified situations it may be less trivial to clearly say when the curvature perturbation is conserved, as, e.g. the effective $\delta P_{\text {nad }}$ may not be negligible when the true matter perturbations are adiabatic. The same could apply to the anisotropic stress.

Previous results on the subject of conserved quantities have not included anisotropic stress [31] and have either done the calculations fully in the large scale approximation [9] or used a different quantity $[11,35,36]$.

\section{Acknowledgements}

PC is funded by a Queen Mary Principal's Research Studentship and by a Bolsa de Excelência Académica of the Fundação Eugénio de Almeida, KAM is supported, in part, by STFC grant ST/J001546/1. The tensor algebra package xAct [37], as well as its sub-package xPand [38, 39], were used in the derivation of many of the equations presented in this work. The authors are grateful to David Mulryne, Adam Christopherson, Timothy Clifton, Julien Larena and Raquel Ribeiro for useful discussions.

\section{A Second order gauge transformations}

Here we present the second order gauge transformations for the quantities used in this paper, given by Eq. (2.21) when applied to the metric and the stress energy tensor. We show only the transformations for the variables defined in the original convention of the metric, Eq. (2.5), as the others can be easily obtained by combining the rules given here with the first order ones in the main text. 
The transformations for the metric quantities are given by

$$
\begin{aligned}
& \widetilde{\psi}_{2}=\psi_{2}-\mathcal{H} \alpha_{2}-\frac{1}{4} \mathcal{X}^{i}{ }_{i}+\frac{1}{4} \nabla^{-2} \mathcal{X}^{i j}{ }_{, i j}, \\
& \widetilde{E}_{2}=E_{2}+\beta_{2}+\frac{3}{4} \nabla^{-2} \nabla^{-2} \mathcal{X}^{i j}{ }_{, i j}-\frac{1}{4} \nabla^{-2} \mathcal{X}^{i}{ }_{i},
\end{aligned}
$$

with $\mathcal{X}_{i j}$ given by (see Ref. [17] for more details)

$$
\begin{aligned}
\mathcal{X}_{i j} \equiv & 2\left[\left(\mathcal{H}^{2}+\frac{a^{\prime \prime}}{a}\right) \alpha_{1}^{2}+\mathcal{H}\left(\alpha_{1} \alpha_{1}^{\prime}+\alpha_{1, k} \xi_{1}{ }^{k}\right)\right] \delta_{i j} \\
& +4\left[\alpha_{1}\left(C_{1 i j}^{\prime}+2 \mathcal{H} C_{1 i j}\right)+C_{1 i j, k} \xi_{1}{ }^{k}+C_{1 i k} \xi_{1}{ }^{k}{ }_{, j}+C_{1 k j} \xi_{1}{ }^{k}{ }_{, i}\right]+2\left(B_{1 i} \alpha_{1, j}+B_{1 j} \alpha_{1, i}\right) \\
& +4 \mathcal{H} \alpha_{1}\left(\xi_{1 i, j}+\xi_{1 j, i}\right)-2 \alpha_{1, i} \alpha_{1, j}+2 \xi_{1 k, i} \xi_{1, j}{ }^{k}+\alpha_{1}\left(\xi_{1 i, j}^{\prime}+\xi_{1 j, i}^{\prime}\right)+\left(\xi_{1 i, j k}+\xi_{1 j, i k}\right) \xi_{1}{ }^{k} \\
& +\xi_{1 i, k} \xi_{1}{ }^{k}{ }_{, j}+\xi_{1 j, k} \xi_{1}{ }^{k}{ }_{, i}+\xi_{1 i}^{\prime} \alpha_{1, j}+\xi_{1 j}^{\prime} \alpha_{1, i} .
\end{aligned}
$$

The transformations for the fluid quantities are

$$
\begin{aligned}
& \widetilde{\delta \rho_{2}}=\delta \rho_{2}+\alpha_{2} \rho^{\prime}+\alpha_{1}\left(\rho^{\prime \prime} \alpha_{1}+\rho^{\prime} \alpha_{1}^{\prime}+2 \delta \rho^{\prime}\right)+\left(2 \delta \rho+\rho^{\prime} \alpha_{1}\right)_{, k}\left(\beta_{1}^{, k}+\gamma_{1}^{k}\right), \\
& \widetilde{\delta P_{2}}=\delta P_{2}+\alpha_{2} P^{\prime}+\alpha_{1}\left(P^{\prime \prime} \alpha_{1}+P^{\prime} \alpha_{1}^{\prime}+2 \delta P^{\prime}\right)+\left(2 \delta P+P^{\prime} \alpha_{1}\right)_{, k}\left(\beta_{1}^{, k}+\gamma_{1}^{k}\right), \\
& \widetilde{v_{2}}=v_{2}-\beta_{2}^{\prime}+\nabla^{-2} \mathcal{X}_{\mathrm{v}}{ }^{k}, k
\end{aligned}
$$

with

$$
\begin{aligned}
\mathcal{X}_{\mathrm{v} i} \equiv & \xi_{1 i}^{\prime}\left(2 \phi_{1}+\alpha_{1}^{\prime}+2 \mathcal{H} \alpha_{1}\right)-\alpha_{1} \xi_{1 i}^{\prime \prime} \\
& -\xi_{1}^{k} \xi_{1 i, k}^{\prime}+\xi_{1}^{k \prime} \xi_{1 i, k}-2 \alpha_{1}\left(v_{1 i}^{\prime}+\mathcal{H} v_{1 i}\right)+2 v_{1 i, k} \xi_{1}^{k}-2 v_{1}^{k} \xi_{1 i, k}
\end{aligned}
$$

\section{B Large scale limit of the gauge invariant quantities}

In this appendix, we supply the expressions for the different versions of the curvature perturbation in the large scale approximation, i.e. when all spatial gradients are taken to be negligible.

The large scale limit of $\zeta_{2}$ and $\zeta_{T 2}$ is given by

$$
\zeta_{2}=\zeta_{T 2}=-\frac{\mathcal{H}}{\rho^{\prime}} \delta \rho_{2}+\frac{1}{\rho^{\prime 2}}\left(2 \mathcal{H}^{2}+\mathcal{H}^{\prime}-\mathcal{H} \frac{\rho^{\prime \prime}}{\rho^{\prime}}\right) \delta \rho_{1}^{2}+\frac{2 \mathcal{H}}{\rho^{\prime 2}} \delta \rho_{1} \delta \rho_{1}^{\prime},
$$

while that for $\zeta_{D 2}$ and $\zeta_{D 2}^{(v)}$ is

$$
\zeta_{D 2}=\zeta_{D 2}^{(v)}=-\frac{\mathcal{H}}{\rho^{\prime}} \delta \rho_{2}+\frac{1}{\rho^{\prime 2}}\left(\mathcal{H}^{\prime}-\mathcal{H} \frac{\rho^{\prime \prime}}{\rho^{\prime}}\right) \delta \rho_{1}^{2}+\frac{2 \mathcal{H}}{\rho^{\prime 2}} \delta \rho_{1} \delta \rho_{1}^{\prime}-\frac{2}{3} h_{1 i j} h_{1}^{i j},
$$

and the limit of $\zeta_{I 2}$ and $\zeta_{I 2}^{(v)}$ is

$$
\begin{aligned}
& \zeta_{I 2}=-\frac{\mathcal{H}}{\rho^{\prime}} \delta \rho_{2}+\frac{1}{\rho^{\prime 2}}\left(\mathcal{H}^{\prime}-\mathcal{H} \frac{\rho^{\prime \prime}}{\rho^{\prime}}\right) \delta \rho_{1}^{2}+\frac{2 \mathcal{H}}{\rho^{\prime 2}} \delta \rho_{1} \delta \rho_{1}^{\prime}-\frac{2}{3} h_{1 i j} h_{1}^{i j}+\frac{1}{3} S_{1 i} S_{1}^{i}, \\
& \zeta_{I 2}^{(v)}=-\frac{\mathcal{H}}{\rho^{\prime}} \delta \rho_{2}+\frac{1}{\rho^{\prime 2}}\left(\mathcal{H}^{\prime}-\mathcal{H} \frac{\rho^{\prime \prime}}{\rho^{\prime}}\right) \delta \rho_{1}^{2}+\frac{2 \mathcal{H}}{\rho^{\prime 2}} \delta \rho_{1} \delta \rho_{1}^{\prime}-\frac{2}{3} h_{1 i j} h_{1}^{i j}+\frac{1}{3} W_{1 i} W_{1}^{i} .
\end{aligned}
$$

These expressions agree with the $\delta N$ formalism, where comparison is possible (see Ref. [9]). 


\section{On intrinsic and extrinsic curvature}

In this appendix, we aim to clarify the relation between the different definitions of $\psi$ and the perturbation to both the intrinsic and extrinsic curvature of hypersurfaces of constant time.

We begin by looking at the intrinsic curvature scalar. It is given by

$$
{ }^{(3)} R=R+R_{\mu \nu} n^{\mu} n^{\nu}-K^{2}+K^{\mu \nu} K_{\mu \nu},
$$

in which $R_{\mu \nu}$ and $R$ are the $4 \mathrm{D}$ Ricci tensor and scalar, respectively, $n^{\mu}$ is the unit normal to the hypersurface, $K_{\mu \nu}$ is the extrinsic curvature and $K$ is its trace. The latter are given by

$$
K_{\mu \nu}=-\frac{1}{2}\left[£_{n} \gamma\right]_{\mu \nu}, \quad K=-\nabla_{\mu} n^{\mu},
$$

with $\gamma$ the induced metric, given by $\gamma_{\mu \nu}=g_{\mu \nu}+n_{\mu} n_{\nu}$. The normal, $n^{\mu}$, is perpendicular to all vectors in the tangent space of the hypersurface. It is therefore often convenient to choose coordinates such that $n_{i}=0$. However, this specific coordinate choice means that the usual gauge transformation rules are not obeyed, and for this reason, we shall also compute these curvature scalars using the 4 -velocity, $u^{\mu}$, to define the spatial hypersurface. In the latter situation, we will denote quantities with the superscript $(u)$.

We now present the calculations of these quantities up to second order in cosmological perturbation theory. The intrinsic curvature scalar is found to be

$$
\begin{aligned}
\delta^{(3)} R_{1} & =\frac{4}{a^{2}} \nabla^{2} \psi_{1}, \\
\delta^{(3)} R_{2} & =\frac{1}{a^{2}}\left[4 \nabla^{2} \psi_{2}-8 C_{1 k m,}^{m} C_{1, n}^{k n}+6 C_{1 m n}{ }^{k} C_{1, k}^{m n}-2 C_{1 k, n}^{k} C_{1 m,}^{m}{ }^{n}\right. \\
& +8 C_{1}^{m n}\left(C_{1 m n, k}{ }_{k}+C_{1 k, m n}^{k}-C_{1 m k, n}{ }^{k}-C_{1 k n, m}{ }^{k}\right) \\
& \left.+4\left(C_{1 k, j}^{k} C_{1, n}^{j n}+C_{1 j k,}{ }^{j} C_{1 m,}^{m}{ }^{k}-C_{1, n, m}^{k} C_{1, k}^{m n}\right)\right],
\end{aligned}
$$

where $C_{i j}$ is the perturbation to the spatial part of the metric. The relation between $\psi$ and the intrinsic curvature is clear at first order, as they are related linearly. This is the reason why the perturbation $\psi$ is called the curvature perturbation. However, this is only true for the original version of $\psi$, as given by the definition (2.5), since all other definitions include a contribution from the metric potential $E$. In any case, at second order, this simple connection between the intrinsic curvature and $\psi$ is lost, as there is no simple relation between any of our definitions of the curvature perturbation and $\delta^{(3)} R_{2}$.

Performing the same calculation using the 4-velocity to define the spatial hypersurface, one finds instead a connection to the curvature perturbation on comoving gauge, $\mathcal{R}$, since the first order result for ${ }^{(3)} R^{(u)}$ is ${ }^{10}$

$$
\delta^{(3)} R_{1}^{(u)}=\frac{4}{a^{2}} \nabla^{2}\left[\psi_{1}-\mathcal{H}\left(v_{1}+B_{1}\right)\right]=\frac{4}{a^{2}} \nabla^{2} \mathcal{R}_{1} .
$$

At second order, however, the result is no longer related to the second order comoving curvature perturbation $\mathcal{R}_{2}$ in a simple way, i.e. $\delta^{(3)} R_{2}^{(u)} \neq \frac{4}{a^{2}} \nabla^{2} \mathcal{R}_{2}$. This can be seen by

\footnotetext{
${ }^{10}$ Note that this quantity has the expected gauge transformation properties, since, being 0 at the background level (because of the assumption of flatness), the Stewart-Walker lemma [40, 41] dictates it to be gauge invariant at first order. Notice that this does not happen in the calculation with $n$.
} 
evaluating $\delta^{(3)} R_{2}^{(u)}$ in comoving gauge $\left(v=B=v_{V}^{i}=0\right)$ and comparing it with $\frac{4}{a^{2}} \nabla^{2} \psi_{2}$. In this gauge, the intrinsic curvature is given by

$$
\delta^{(3)} R_{2}^{(u)}=\delta^{(3)} R_{2}+S_{1}^{i} w_{i}+S_{1}^{i, j} w_{i j},
$$

where $w_{i}$ and $w_{i j}$ are linear functions of the metric potentials. It is clear that this is not equal to $\frac{4}{a^{2}} \nabla^{2} \psi_{2}$, as there are no further cancellations that would recover that result. Therefore, one must conclude that none of our definitions of $\psi$ has a straightforward interpretation as the perturbation to the intrinsic curvature at an order higher than first.

Moving now to the scalar extrinsic curvature, we start by noting that it is proportional to the local expansion $\nabla_{\mu} n^{\mu}$ (or $\nabla_{\mu} u^{\mu}$, when choosing the velocity 4 -vector to define the spatial hypersurface). It is well known that the integral of the expansion along world lines, with respect to proper time $s$, can be used to define a local scale factor $[9,11]$. This integral is defined as

$$
\alpha=\frac{1}{3} \int \nabla_{\mu} n^{\mu} d s=-\frac{1}{3} \int K d s,
$$

and the local scale factor is given by $e^{\alpha}$. This interpretation is further supported by the fact that, at the background level, one has $\alpha^{\prime}=\mathcal{H}$. At first order, one finds

$$
\delta \alpha_{1}^{\prime}=-\psi_{1}^{\prime}-\frac{1}{3} \nabla^{2}\left(B_{1}-E_{1}^{\prime}\right)
$$

This variable has some similarity with our definition of $\psi_{T}$, but still has a contribution from $B$, which is not present in any of our versions of the curvature perturbation at first order. Turning now to the situation with $u$ as the normal vector, the first order result is

$$
\delta \alpha_{1}^{(u) \prime}=-\psi_{1}^{\prime}+\frac{1}{3} \nabla^{2}\left(v_{1}+E_{1}^{\prime}\right) .
$$

While this is still not equal to any version of $\psi$ directly, $\delta \alpha_{1}^{(u)}$ is, in fact, equal to $\zeta_{1 T}$, when the latter is evaluated using a uniform density slicing. Going to second order, we find

$$
\begin{aligned}
\delta \alpha_{2}^{(u) \prime}= & -\psi_{2}^{\prime}+\frac{1}{3} \nabla^{2}\left(v_{2}+E_{2}^{\prime}\right)+\frac{1}{3}\left(-4 C_{1 i j} C_{1}^{i j \prime}+2 \phi_{1} \nabla^{2} v_{1}+2\left(v_{V 1}^{i}+v_{1}^{, i}\right)\left(\phi+C_{1 j}^{j}\right)_{, i}\right. \\
& \left.+\left[\left(v_{V 1}^{i}+v_{1}^{i}+B_{1}^{, i}-S_{1}^{i}\right)\left(v_{V 1 i}+v_{1, i}+B_{1, i}-S_{1 i}\right)\right]^{\prime}\right) \\
& -2\left(v_{V 1}^{i}+v_{1}^{, i}\right)\left(-\psi_{1}+\frac{1}{3} \nabla^{2} \int\left(E_{1}^{\prime}+v_{1}\right) d \tau\right)_{, i} .
\end{aligned}
$$

Again, this variable is not equal to any version of $\psi$, but it becomes exactly $\psi_{I}$, when evaluated using a comoving threading $\left(v=v_{V}^{i}=0\right)$. This is equivalent to saying that, by applying the same procedure to this quantity, one would obtain a gauge invariant quantity that is equal to $\zeta_{I 2}^{(v)}$. This is not surprising, given the results of Refs. [11,31, 35, 36], which found similar evolution equations for gauge invariants defined from the expansion scalar, $\Theta=\nabla_{\mu} u^{\mu}$.

We conclude our exposition of this appendix by noting that, even though the connection between the intrinsic curvature and $\psi$ is lost at second order, it is still possible to find a definition of $\psi$ which closely matches the extrinsic curvature scalar for comoving hypersurfaces (i.e. space-like hypersurfaces which are normal to the velocity 4-vector), at least when evaluated in a particular gauge. The reason why the version of $\psi$ that resembles $K$ is the one 
arising from the determinant of $g^{i j}$ can be explained by a relation between the determinant of the metric and the covariant divergence of a 4 -vector. This is given by

$$
\nabla_{\mu} u^{\mu}=\partial_{\mu} u^{\mu}+\Gamma_{\nu \mu}^{\mu} u^{\nu}=\partial_{\mu} u^{\mu}+u^{\nu} \partial_{\nu} \log (\sqrt{-g}),
$$

in which $g=\operatorname{det}\left[g_{\mu \nu}\right]$. Furthermore, it can be shown that $\operatorname{det}\left[g^{i j}\right]$ is related to $g$ by

$$
g=g_{00}\left(\operatorname{det}\left[g^{i j}\right]\right)^{-1},
$$

and thus the previous relation becomes

$$
\nabla_{\mu} u^{\mu}=\partial_{\mu} u^{\mu}+u^{\nu} \partial_{\nu} \log \left(\sqrt{-g_{00}}\right)-u^{\nu} \partial_{\nu} \log \left(\sqrt{\operatorname{det}\left[g^{i j}\right]}\right) .
$$

Choosing a comoving threading is equivalent to setting $u^{i}=0$ and in that case it is straightforward to show that $u^{0}=\sqrt{-g_{00}}$. This implies that the first two terms on the r.h.s. of Eq. (C.13) cancel, and one finds

$$
\left(\nabla_{\mu} u^{\mu}\right)_{\mathrm{com}}=-u^{\nu} \partial_{\nu} \log \left(\sqrt{\operatorname{det}\left[g^{i j}\right]}\right)=3 \frac{d}{d s}\left(\log a+\psi_{I}\right),
$$

in which we substituted $\operatorname{det}\left[g^{i j}\right]$ by the definition of $\psi_{I}$. Equivalently, one has

$$
-\left(\frac{1}{3} \int K^{(u)} d s\right)_{\mathrm{com}}=\log a+\psi_{I} .
$$

This shows $\psi_{I}$ to be the perturbation to the integrated extrinsic curvature of comoving hypersurfaces when written using a comoving threading. This result is valid at all orders and provides a clear interpretation to this perturbation derived from the determinant of the spatial part of the inverse metric.

\section{References}

[1] Hinshaw G et al. (WMAP) 2013 Astrophys.J.Suppl. 20819 (Preprint 1212.5226)

[2] Ade P A R et al. (Planck) 2015 (Preprint 1502.01589)

[3] Ade P et al. (BICEP2, Planck) 2015 Phys. Rev. Lett. 114101301 (Preprint 1502.00612)

[4] Bardeen J M, Steinhardt P J and Turner M S 1983 Phys. Rev. D28 679

[5] Salopek D S and Bond J R 1990 Phys. Rev. D42 3936-3962

[6] Acquaviva V, Bartolo N, Matarrese S and Riotto A 2003 Nucl. Phys. B667 119-148 (Preprint astro-ph/0209156)

[7] Bartolo N, Komatsu E, Matarrese S and Riotto A 2004 Phys. Rept. 402 103-266 (Preprint astro-ph/0406398)

[8] Wands D, Malik K A, Lyth D H and Liddle A R 2000 Phys. Rev. D62 043527 (Preprint astro-ph/0003278)

[9] Lyth D H, Malik K A and Sasaki M 2005 JCAP 0505004 (Preprint astro-ph/0411220)

[10] Rigopoulos G and Shellard E 2003 Phys.Rev. D68 123518 (Preprint astro-ph/0306620)

[11] Langlois D and Vernizzi F 2005 Phys.Rev. D72 103501 (Preprint astro-ph/0509078) 
[12] Malik K A and Wands D 2004 Class. Quant. Grav. 21 L65-L72 (Preprint astro-ph/0307055)

[13] Tomita K 1967 Progress of Theoretical Physics 37 831-846 (Preprint http://ptp. oxfordjournals.org/content/37/5/831.full.pdf+html) URL http://ptp.oxfordjournals.org/content/37/5/831.abstract

[14] Tomita K 1971 Progress of Theoretical Physics 45 1747-1762 (Preprint http://ptp.oxfordjournals.org/content/45/6/1747.full.pdf+html) URL http://ptp.oxfordjournals.org/content/45/6/1747. abstract

[15] Tomita K 1972 Progress of Theoretical Physics 47 416-443 (Preprint http://ptp.oxfordjournals.org/content/47/2/416.full.pdf+html) URL http://ptp.oxfordjournals.org/content/47/2/416. abstract

[16] Matarrese S, Mollerach S and Bruni M 1998 Phys. Rev. D58 043504 (Preprint astro-ph/9707278)

[17] Malik K A and Wands D 2009 Phys.Rept. 475 1-51 (Preprint 0809.4944)

[18] Mukhanov V F, Feldman H A and Brandenberger R H 1992 Phys. Rept. 215 203-333

[19] Bardeen J M 1980 Phys.Rev. D22 1882-1905

[20] Kodama H and Sasaki M 1984 Prog.Theor.Phys.Suppl. 78 1-166

[21] Maldacena J M 2003 JHEP 05013 (Preprint astro-ph/0210603)

[22] Ellis G F R, Maartens R and MacCallum M A H 2012 Relativistic Cosmology (Cambridge University Press)

[23] Noh H and Hwang J c 2003 (Preprint astro-ph/0305123)

[24] Malik K A and Matravers D R 2008 Class. Quant. Grav. 25193001 (Preprint 0804.3276)

[25] Malik K A and Matravers D R 2013 Gen.Rel.Grav. 45 1989-2001 (Preprint 1206.1478)

[26] Christopherson A J, Nalson E and Malik K A 2015 Class.Quant.Grav. 32075005 (Preprint 1409.5106)

[27] Dias M, Elliston J, Frazer J, Mulryne D and Seery D 2015 JCAP 1502040 (Preprint 1410.3491)

[28] Mollerach S 1990 Phys. Rev. D42 313-325

[29] Christopherson A J and Malik K A 2009 Phys. Lett. B675 159-163 (Preprint 0809.3518)

[30] Leithes A and Malik K A 2015 Class.Quant.Grav. 32015010 (Preprint 1403.7661)

[31] Enqvist K, Hogdahl J, Nurmi S and Vernizzi F 2007 Phys. Rev. D75 023515 (Preprint gr-qc/0611020)

[32] D'Amico G, de Rham C, Dubovsky S, Gabadadze G, Pirtskhalava D and Tolley A J 2011 Phys. Rev. D84 124046 (Preprint 1108.5231)

[33] De Felice A, Gmrkolu A E, Mukohyama S, Tanahashi N and Tanaka T 2014 JCAP 1406037 (Preprint 1404.0008)

[34] Fasiello M and Ribeiro R H 2015 JCAP 1507027 (Preprint 1505.00404)

[35] Langlois D and Vernizzi F 2006 JCAP 0602014 (Preprint astro-ph/0601271)

[36] Langlois D and Vernizzi F 2007 JCAP 0702017 (Preprint astro-ph/0610064)

[37] Martín-García J M 2008 Computer Physics Communications 179 597-603 (Preprint 0803.0862)

[38] Brizuela D, Martin-Garcia J M and Mena Marugan G A 2009 Gen.Rel.Grav. 41 2415-2431 (Preprint 0807.0824) 
[39] Pitrou C, Roy X and Umeh O 2013 Class.Quant.Grav. 30165002 (Preprint 1302.6174)

[40] Stewart J M and Walker M 1974 Proc. Roy. Soc. Lond. A341 49-74

[41] Stewart J M 1990 Class. Quant. Grav. 7 1169-1180 